ferat zugänglich war. Letzterer Autor beschreibt nehmlich an der hinteren Fläche der Wurzel des Kehldeckels eine quere Falte, welche eine nach oben oflene Tasche bildete; der untere befestigte Rand dieser Tasche ging in einen dreieckigen Wulst über, welcher mit einem unteren spitzen Winkel zwischen die oberen Stimmbänder hineinragte. Die Lage der Hyrtl'schen Tasche entspricht also genau der Lage des Sinus praeepiglotticus des Pavians: denkt man sich die Tasche weiter nach vorne vertieft, so müsste sie ganz wie der letztere die Knorpelsubstanz der Epiglottis von hinten nach vorne perforiren und sich je nach ihrer Ausdehnung vor der vorderen Epiglottisfläche in Form eines grösseren oder kleineren Sackes ausbreiten. Mit meinem Fall ist der Hyrtl'sche jedenfalls nicht in eine Kategorie zu stellen, weil die von Hyrtl beschriebene Tasche eben nicht zwischen den vorderen Enden der Stimmbänder, sondern über denselben an der hinteren Epiglottisfläche gelegen war.

Bei anderen Affen, die ich darauf hin untersuchte, wie z. B. einem Kapuciner, Orang-Utang und Gorilla, war weder von einem Sinus sub- noch von einem Sinus praeepiglotticus eine Andeutung wahrzunehmen, so dass also die letztere Form von Divertikel dem Pavian eigenthümlich zu sein scheint. Ebenso wenig sind diese Ausbuchtungen der Larynxschleimhaut bei den anderen Haussäugethieren mit Ausnahme der Einhufer vorhanden. Das eben beschriebene Präparat ist der Sammlung des hiesigen anatomischen Instituts einverleibt und deswegen von mir nicht abgebildet worden, wiewohl ich nicht im Stande war, einen ähnlichen Fall in der Literatur aufzufinden.

\title{
III. Deber einen Fall von Divertikel der Seitenwand des Pharynx in Communication mit der Tuba Eustachii.
}

Im vergangnen Wintersemester fand ich an der rechten Hälfte eines median durchschnittenen menschlichen Kopfes eine auffallend tiefe Rosenmüller'scher Grube vor; als ich die letztere weiterhin genauer untersuchte, entdeckte ich hinter dem hintern Theil der Tubenmündung einen schmalen schlitzförmigen Spalt 
von etwa $7 \mathrm{~mm}$ Länge, in welchen man bequem eine Sonde bis zu einer Tiefe von $2 \mathrm{~cm}$ einfübren konnte. Die Höhle des hier vorhandenen Divertikels erstreckte sich nach lateralwärts hin und liess sich mit einer ziemlich beträchtlichen Menge von Watte ausstopfen. Da sich nun beim Pferde bekanntlich in Gestalt der sog. Luftsäcke (poches gutturales der Franzosen) kolossale Ausstülpungen der Tuba Eustachii vorfinden, welche sich nach oben bis an die Schädelbasis, nach unten bis an das Zungenbein, Jateralwärts bis an Hals- und Kaumuskeln erstrecken, und einander medianwärts berühren, so versuchte ich auch die Tube zu sondiren und konnte in der That ohne Anwendung von Gewalt und ohne gebohrt zu haben, die Sonde durch die Tubenmündung soweit nach hinten führen, dass der Knopf derselben in dem eben beschriebenen Spalt der Rosenmüller'schen Grube sichtbar wurde. Ich ging nun daran das Divertikel präparatorisch von aussen blosszulegen; zu diesem Zweck wurde der Jochbogen durchsägt und mit dem Musc. masseter nach abwärts geschlagen, hierauf der Processus coronoideus des Unterkiefers abgetragen und mit dem Musc. temporalis nach oben zurückgelegt. Nachdem dann noch der Unterkiefer vorsichtig exarticulirt und nach abwärts gezogen war, eröffnete ich einen HobJraum zu welchem man mit der Sonde von dem oben beschriebenen Schlitz hinter der Tubenmündung aus gelangen konnte. Die Wand dieses Hohlraums war innen ganz glatt, sehr dünn und leicht zerreislich, was allerdings zum Theil wohl darin seine Erklärung findet, dass der betreffende Kopf schon etwa 14 Tage in dem Secirsaal gelegen hatte, bevor ich das Divertikel bemerkte und in Präparation nahm. Das Letztere erstreckte sich nach lateralwärts zwischen dem Proc. coronoideus des Unterkiefers und den vom Proc. styloideus ausgehenden Muskeln bis an die Parotis; vorne grenzte es an dic Musc. pterygoidei ext. und int., sowie an das Lig. laterale int. des Kiefergelenks; hinten an den Proc. styloideus, die von demselben ausgehenden Muskeln und Bänder und an die Carotis int.; nach oben reichte die Möhle bis an die Spina angularis und an die Pars tympanica des Schläfenbeins, insoweit die letztere nicht durch das Kiefergelenk eingenommen ist. Nach unten erstreckte sich das Divertikel bis etwa zur Nitte des Kieferastes und medianwärts war seine Oeffnung in den Pharynx 
umgrenat von dem Musc. levator veli palatini und dem Constrictor pharyngis sup. Der Zugang zu dem Divertikel vom Pharynx ans bildete einen kurzen Gang, eine Art Vorraum von etwa $5 \mathrm{~mm}$ Höhe und $1 \mathrm{~cm}$ Länge, und wenn ich eine Sonde in die Tubenmündung nach hinten einführte, kam ich mit dem Knopf derselben zwischen den Musc. petro- und sphenostaphylinus an derjenigen Stelle heraus, an welcher der eben erwähnte Vorraum in die beschriebene Haupthöhle des Divertikels einmündete. Dieser Vorraum lag nun über den obersten Fasern des M. constrictor pharyngis sup. d. h. das Divertikel hatte sich an derjenigen Stelle der Seitenwand des Schlundkopfs ausgebuchtet, an welcher der letztere nicht mehr musculöse Wände besitzt. Bei mikroskopischer Untersuchung liess sich an der Innenwand des Hohlraums keinerlei Epithel mehr nachweisen; es kann dies kaum befremden, wenn man erwägt, dass die Wände desselben bei den Kan- und Schluckbewegungen stetem Druck und steten Verschiebungen gegen einander ausgesetzt sein mussten und somit die Epithelauskleidung wahrscheinlich schon intra vitam auf diese Weise verloren gegangen sein wird. Es ist indess auch möglich, dass ihr Fehlen auf die cadaverösen Veränderungen zurückzuführen ist, welche immerhin in gewissem Grade an dem Präparat Platz gegriffen hatten.

Irgend ein besonderer Inhalt war in der Höhle nicht vorhanden. Leider war es mir nicht möglich, die-sondirte Communication des Hohlraums mit der Tube herauszupräpariren; sie ging mir in dem leicht zerreisslichen Gewebe völlig verloren, ebenso wie ich es auch nicht vermeiden konnte die Wand des Divertikels anzuschneiden. Nachdem ich dann noch die Tube nach oben hin aufgeschnitten hatte, konnte ich an der hintern und untern Wand derselben entsprechend der Rüdinger'schen Hülfsspalte eine deutliche Vertiefung und in derselben einen deutlichen Schlitz constatiren, durch welchen die Sonde bis in das Divertikel gelangt sein musste. Jedenfalls bin ich äberzeugt, mich nicht auf künstlichen Wegen befunden zu haben, da die Wand der Tube für gewöhnlich durchaus nicht so leicht zerreisslich ist, und sich selbst an faulen Leichen nur unter Anwendung von Gewalt perforiren lässt. Auch findet sich normaler Weise nicht, wie in unserem Fall an der Stelle des Schlitzes 
eine so ausgeprägte Vertiefung der Tubenwand, die mit einem hier vorhanden gewesenen Divertikel gut in Einklang zu bringen ist.

Von weitern Besonderheiten an dem vorliegenden Präparate möchte ich noch betonen, dass die Mandeln ganz auffallend tiefe folliculäre Recessus besassen, in welehe sich die Sonde bis zu $1,5 \mathrm{~cm}$ einführen liess, und welche in der Tiefe mit einander communicirten. An der Stelle des Canalis incisivus fand sich eine anscheinend mit Schleimhaut ausgekleidete Cyste, welche durch den Medianschnitt eröffnet worden war. Leider war die entsprechende linke Kopfhälfte bereits weggeworfen, so dass sich nicht mehr constatiren liess, ob hier ähnliche Abnormitäten wie rechts vorhanden gewesen waren.

Kleinere Divertikel des Pharynx gehören keineswegs zu den Seltenheiten. Wie wir an der Zungenwurzel die sog. Zungentonsille und an der Seitenwand des Isthmus fautium die sog. Mandel oder Rachentonsille als die beiden bekanntesten grösseren Anhäufungen von lymphatischen Balgdrüsen oder Follikeln vorfinden, so haben wir weitere derartige Lymphapparate bekanntlich noch zwischen der untern Tubenmündung und dem Gaumen in Gestalt der Tubentonsille und zwischen den beiden Rosenmüller'schen Gruben als retropharyngeale Tonsille (PharynxTonsille der Autoren), so dass sich also von der Zungenwurzel an bis zum obern Theile der hintern Pharynxwand ein nahezu continuirlicher lymphatischer Rachenring hinzieht. An allen Punkten dieses Rachenringes finden sich nun die bekannten grössern oder kleinern, mitunter nur mikroskopischen folliculären Recessus oder Lacunen, wie sie an den Mandeln am constantesten vorkommen und sich hier mitunter recht tief in die Schleimhaut einsenken, insbesondere wenn sie intra vitam durch stagnirende Käsepfröpfe ausgedehnt waren. Besonders häufig kommt ausserdem in der Medianlinie der obern hintern Rachenwand, d. h. also in der Mitte der Pharynxtonsille die Mayer'sche ${ }^{1}$ ) Bursa pharyngea vor, welche nach Luschka eine bis $15 \mathrm{~mm}$ lange, $6 \mathrm{~mm}$ breite gegen die Pars basilaris gerichtete Vertiefung des Pharynxgewölbes bildet und eine im Mittel $1 \mathrm{~mm}$ dicke Wand

1) Mayer, Neue Untersuch. aus d. Geb. der Anat. und Phys. Bonn 1842. 
aus überwiegend adenoiden Gewebe besitzt. Ueber die Bedeutung dieses Divertikels sind die Autoren noch keineswegs einig. Luschka') und Landzert ${ }^{2}$ ) betrachten die Bursa als einen erweiterten Rest des inconstanten Canalis cranio-pharyngeus, welcher von der Fossa sellae turcicae durch den mittleren Keilbeinkörper als fibröser gewöhnlich hohler Fortsatz hinzieht. Frorie ${ }^{3}$ ) bringt die Bursa mit dem vordern Ende der Chorda dorsalis in Verbindung, welches zu einer gewissen Zeit in dem retropharyngealen Bindegewebe gelegen ist und die angrenzende Stelle der Pharynxwand bei dem weitern Schädelwachsthum gewissermaassen trichterförmig nach hinten zieht. Henle ${ }^{4}$ ) endlich scheint die Bursa pharyngea lediglich für einen abnorm grossen folliculären Recessus zu halten und ist damit vielleicht der Wahrheit am nächsten gekommen; er giebt auch an, dass derartige Recessus der hintern und obern Rachenwand bald nur seichte Vertiefungen darstellen, an deren Grund sich die Mündungen secundärer Autstülpungen vorfinden, bald in der Form einer einfachen oder mehrerer Blasen auftreten und sich bis in das knorpelharte Bindegewebe der Schädelbasis erstrecken können.

Eine andere Kategorie von Pharynxdivertikeln ist offenbar mit den sog. Fistulae colli congenitae in eine Reihe zu stellen. Die Oeffnungen dieser Fisteln führen entweder von aussen hin zum Larynx, der Trachea oder dem Pharynx hin, oder endigen blind im Bindegewebe des Halses.

In einem Fall von Neuhöfer ${ }^{5}$ ) mündete der rechte Fistelgang am hintern Rande des Musc. pharyngo-palatinus, da wo dieser in den Pharynx ïbergeht; der linke erstreckt sich etwas weiter nach oben. Stellt man sich nun an einer solchen Fistula colli congenita das äussere, der Haut des Halses näher gelegene Ende obliterirt vor, so erhält man einen Zustand, wie er von Watson ${ }^{6}$ ) an einem sehr interessanten Fall beschrieben worden

1) Luschka, Schlundkopf des Menschen. Tübingen 1868.

2) Landzert, Ueber den Canalis eranio phar. St. Petersburger med. Zeitschrift. Bd. XIV. 1868.

3) Froriep, Kopftheil der Chorda bei menschlichen Embryonen, Festgabe von Henle's Schülern 388 ?.

4) Henle, Handb. d. Anat. 1873. Bd. II. S. 90.

5) Neuhöfer, Bayrisches Correspondenzbl. 1857.

6) Watson, Journ. of anat. and phys. London and Cambridge. May 1875. 
ist. Im oberen carotischen Dreieck unter der Sehne des Musc. digastricus wurde ein langer, unten sackförmig erweiterter Schlauch sichtbar, der sich dicht unter den Fascien und der Haut des Halses bis zum Manubrium sterni hinuntererstreckte. Der Stiel des Sackes verlief über dem Musc. stylopharyngeus und dem Nervus hypoglossus gegen den Isthmus faucium, wo er dicht hinter der Tonsille am freien Rand des hintern Gaumenbogens mit einer schmalen schlitzförmigen Oeffnung mündete. Die Wand des Divertikels war mit quergestreiften Muskeln, Blutgefässen und Nervenzweigen von Seiten des Glossopharyngeus reichlich versehen.

Mit Recht verwirft Watson unter den vorliegenden Verhältnissen den Gedanken an eine postfötale Ausbuchtung durch Druck, an ein sog. Pulsionsdivertikel und denkt an die Möglichkeit des Offenbleibens der ersten postmandibularen Visceralspalte, ohne im übrigen die Schwierigkeiten zu verkennen, welche sich einem detaillirten Eingehen in die Genese desselben entgegenstellen.

Ungefähr zu derselben Zeit veröffentlichte Zuckerkandl') einen anderu Fall von Pharynxdivertikel unter dem Namen eines Recessus salpingopharyngeus. An beiden Seitenwänden des Cavum pharyngo-nasale fanden sich die schon an und fïr sich bedentend entwickelten Mündungen der Tuben in Form von etwa $1,5 \mathrm{~cm}$ langen und breiten Gruben bis an die obere Fläche des weichen Gaumens fortgesetzt. Die Gruben waren nach vorn durch die sog. Plica salpingopalatina (Tourtual) begrenzt, die, gestüzt von dem gleichnamigen Bande, von der Tubenmündung zur sehnigen Ausbreitung des Gaumenhügels verläuft und etwa dem vordern Rand des Tensor veli palatini entspricht; hinten war ibre Grenze durch einen Wulst gebildet, welcher durch den darunter befindlichen Musc. levator palati mollis hervorgedrängt wurde; der Recessus senkte sich also zwischen dem abnorm weit auseinandergewichenen Heber und Spanner des Gaumens in die Seitenwand des Pharynx ein bis zu dem Fascienblatt, welches diese Muskeln von aussen bedeckt. Eine zweite halbmondförmige Falte theilte den Recessus in eine vordere flache und hintere

1) Zuckerkandl, Monatsschrift für Ohrenheilkunde. 1875. No. 2. 
tiefe Grube. Uebrigens macht die beigegebene Zeichnung durchaus den Eindruck, als ob ein Theil des membranösen Tubenbodens mit in den Bereich der Vertiefung gezogen wäre und als ob der Knorpel der Tube trotz seiner kräftigen Entwicklung kürzer wie in der Norm gewesen wäre. Ueber die Genese dieser Vertiefungen spricht sich Z u ckerkandl nicht aus, doch scheint er jedenfalls die Bildung für congenital zu halten; er glaubt, dass sich zwischen der Plica salpingopharyngea und dem Musc. levator palati bei einer gewissen Entwicklung derselben stets eine Grube befände, welche in seinem Falle lediglich sehr stark vertieft gewesen wäre.

Endlich ist in neuester Zeit von Pertik') ein Fall von doppelseitigem Divertikel des Pharynx veröffentlicht worden. Die bilateral symmetrischen Säcke buchteten sich nach unten und hinten von der Tubenmündung an der seitlichen und anscheinend auch an der hintern Rachenwand aus. Ihre Lage war vorne durch den hintern Rand des kräftig entwickelten Levator palati mollis, hinten und unten durch den concaven obern Rand des M. cephalopharyngeus begrenzt. Der Zugang zu den Diverticeln war beträchtlich weit und bildete ein Oval von etwa 16 bis $18 \mathrm{~mm}$ Länge und 12-14 mm Breite; die Tiefe der Säcke betrug etwa $15 \mathrm{~mm}$. Pertik macht mit Recht darauf aufmerksam, dass es zwei Puncta minoris resistentiae an der Pharynxwand gäbe, an welchen die letztere nicht durch Muskeln geschützt sei; von diesen beiden Punkten entspricht der cine dem Zuckerkandl'schen Recessus salpingopharyngeus und ist zwischen dem Levator und Tensor veli gelegen, der zweite Punkt würde durch das Pertik'sche Divertikel und seine eben beschriebenen Grenzen bezeichnet sein. Pertik hält nun seinen Fall für einen Pulsionsdivertikel, d. h. er glaubt, dass dasselbe entstanden sei durch den Druck heftiger nasaler Exspirationen und der Luftcompression bei Schluckbewegungen, durch welche zuerst diese weniger resistente, membranöse Stelle der Rachenwand ausgedehnt werden müsste. Den Zuckerkandl'schen Fall hält $P$. jedoch trotz dieser Auffassung für eine angeborne Form der Ausstälpung. Wenngleich er nun für seine Ansicht 
betreffs des von ihm beschriebenen Objectes sehr wichtige Gründe anführt, so möchte ich doch glauben, dass auch hier ursprünglich eine, wenn auch nur geringe, congenitale Ausbuchtung vorhanden gewesen sein mag, welche durch die von ihm betonten Momente weiterhin vergrössert wurde. Andernfalls müssten doch dergleichen Pulsionsdivertikel häufiger vorkommen, da chronische Rachen- und Nasenkatarrhe mit vollständiger Impermeabilität der Nase keineswegs zu den Seltenheiten gehören, während dagegen der Pertik'sche Fall bisher anscheinend noch nicht beschrieben worden ist.

Das von mir aufgefundene Divertikel lässt sich offenbar mit allen eben erwähnten kaum in eine Kategorie stellen. Die eine in der Rosenmüller'schen Grube befindliche Mündungsöffnung würde der Lage nach mit dem Pertik'schen Falle übereinstimmen; der andere in der Tube befindliche Zugang würde sich an den Zuckerkandl'schen Recessus anschliessen, wenn man erwägt, dass bei letzterem Fall jedenfalls ein Theil des Tubenbodens in den Bereich des Recessus einbezogen war. Die Frage nach der Genese kann ich vun für die von mir beschriebene Bildung nur hypothetisch und in beschränkter Weise erörtern. Doch möchte ich darauf hinweisen, dass wir es hier möglicher Weise mit einer interessanten Theromorphie zu thun haben, welche in den schon erwähnten Luftsäcken der Einhufer ihr Aualogon findet und soviel mir bekannt bei andern Thieren nicht vorhanden ist. Diese colossalen Luftsäcke, über welche ich mich beim Pferde orientiren konnte, stellen eine Fortsetzung der Schleimhaut der Tuba Eustachii vor und hängen durch einen Schlitz in der Wand der letztern mit dem Lumen derselben zusammen. Ueber die eigentliche Bedeutung dieser Luftsäcke ist man auch für die letzgenannten Thiere keineswegs im Klaren. Nach einzelnen Autoren sollen dieselben eine schnelle Erneuerung der Luft in der Trommelhöhle möglich machen, nach Andern wie ein elastisches Kissen für den schweren Kopf, nach einer dritten Ansicht chemischen Respirationszwecken dienen. Noch eine Anzahl weiterer Hypothesen sind hierüber aufgestellt worden. Indessen wie dem auch sei - wenn ich das von mir gefundene Divertikel der Tube als Theromorphie bezeichne, so ist damit doch keineswegs gesagt, dass demselben zu gleicher Zeit 
eine atavistische Bedeutung beizumessen sei. Dies muss auch für den von mir vorhin geschilderten Ventriculus laryngis tertius festgehalten werden. Die Aehnlichkeit zwischen einer abnormen menschlichen und einer normalen thierischen Bildung könnte immerhin eine zufällige, durch äussere accidentelle Gründe bedingte sein. Dennoch wird man, glaube ich, zur Zeit gutthun, auf eine jede derartige Aehnlichkeit hinzuweisen und sie solange als bemerkenswerth zu bezeichnen, als man für die Genese dieser Anomalien keine genügende Erklärung besitzt.

Ich bin also geneigt anzunehmen, dass das von mir be-. schriebene Divertikel als eine primäre Ausstülpung des membranösen Theils der Tuba Eustachii zu betrachten sei. Immerhin bliebe dann die weitere Frage zu erörtern, wie diese Ausstülpung durch den in der Rosenmüller'schen Grube befindlichen Schlitz mit dem Pharynx in Communication getreten ist. Dies möchte ich in der Weise erklären, dass sich ursprünglich an der Stelle dieses Schlitzes ein stark entwickelter folliculärer Recessus befunden hat, welcher später mit dem Tubendivertikel unter beiderseitiger Verdünnung der Wände verschmolzen ist. Hierfür scheint mir auch der Umstand zu sprechen, dass der ebenerwähnte Schlitz erst zu einem etwa $1 \mathrm{~cm}$ langen Vorraum führte, welcher in die eigentliche Haupthöhle des Divertikels einmündete und etwa die Beschaffenheit eines tiefen folliculären Recessus besass. Ich habe schon erwähnt, dass solche Recessus an dieser Stelle keine Seltenheit vorstellen, und dass auch die Mandeln bei demselben Kopf durch dergleichen tiefe Buchten ausgezeichnet waren. Jedenfalls kann ich mich bei einem Vergleich mit dem Pertik'schen Fall nicht dazu entschliessen, den meinigen als Pulsionsdivertikel der Rosenmüller'schen Grube aufzufassen, wogegen auch die schmale spaltförmige Configuration der Oeffnung spricht, welche überhaupt leicht der Wahrnehmung entgehen konnte. Ebensowenig kann ich meinen Fall als Ueberrest einer unverschlossen gebliebenen Visceralspalte deuten. Das betreffende Präparat ist in der Sammlung des hiesigen anatomischen Instituts aufgehoben worden und habe ich es aus diesem Grunde nicht für nöthig gehalten eine diesbezügliche Abbildung beizugeben. 